\title{
The Role of Music Therapy in Promoting Communication and Social Skills in Children with Autism Spectrum Disorder: A Pilot Study
}

\author{
Monica Subiantoro \\ Universitas Pelita Harapan, Tangerang \\ monica.subiantoro@uph.edu
}

\begin{abstract}
Based on data gathered by WHO, 1 in 160 children has autism (WHO, 2017). This rapidly increasing number has alarmed parents and healthcare professionals. One of the primary concerns raised by parents of children with autism spectrum disorder, or ASD, is the lack of initiative from their children in communication. Prior to verbal means of communication, parents communicate with their babies using musical cues such as rhythm, dynamics, melody, volume, tempo, and intensity (Stern, 1985). In addition, 'motherese' or infant-directed singing is found effective in promoting parent-infant bonding (Gardner \& Goldson, 2002). The abovementioned theories support the practice of music therapy on children with communication issues. Given the growing practice with this population worldwide (Wigram, 2002; Oldfield, 2006; Thompson, 2012), music therapy is considered a new intervention in Indonesia. This pilot study aims to investigate the effects of music therapy in communication and social skills development of a child with autism. It includes a single-case study that has been analyzed qualitatively through observation and video analysis. A child received individual weekly music therapy sessions for a year at home, run by a registered music therapist. The results showed an increase of eye contact, turntaking, and shared playing. The enjoyment in music making and improvisation leads to the awareness of other's presence in the room, which later translates into the responsiveness in both musical and verbal cues.
\end{abstract}

Keywords: Music, therapy, children, autism, communication.

\section{INTRODUCTION}

According to WHO (2017), 1 in 160 children has autism. This striking ratio has motivated the emergence of various studies in early diagnosis and intervention, including music therapy. In music therapy, music and its elements are utilized as non-verbal means of communication to achieve non-musical goals. Given the limitations of verbal communication in many children with Autism Spectrum Disorder (ASD), music therapy becomes a potentially suitable intervention for this population.

Music therapy with autism has a long history, starting in the 1940, when autism was first considered as a special disorder (Holck, 2011). From documented and published case studies, to randomized controlled trials conducted by music therapy researchers, research on music therapy and ASD children in a variety of designs evolved and further strengthened the practice of music therapy in this area, especially in countries where music therapy has been an established profession and offered as a nonpharmacological intervention.

Earlier this year, TIME-A project, the biggest randomized controlled trial in music and autism which involved 364 children with autism aged 4-7 in 9 countries, published a result that music therapy does not show a significant effect in reducing symptoms in autism compared to the standard care (CNN, 2017). Although this study is considered strong, given its large sample size, different cultural backgrounds in each participating country as well as methods used in intervention have been considered as the challenges in this research.
Since music therapy is a culturally sensitive practice, research on its practice needs to be conducted in Indonesia. Given the increasing public awareness of autism and this nation's potentials in music and arts, the present study is expected to explore the use of music as a therapeutic tool in developing social interaction and communication of children with ASD in the context of our local culture, as well as providing a wider insight into music therapy as a discipline. Ultimately, it is hoped that music therapy can contribute to the improvement of health and wellbeing in our society, especially children with autism spectrum and their families.

\section{LITERATURE REVIEW}

\begin{abstract}
Autism
Autism is a neurodevelopmental disorder that is usually noticed and diagnosed in a 2-3 year old child. According to the Diagnostic and Statistical Manual of Mental Disorders-5 (DSM-5), the criteria used to diagnose autism consists of lack of communication and social interaction, restricted and repetitive behavioral patterns, the appearance of symptoms from an early age, limiting symptoms and disrupting day-to-day functions, and existing symptoms cannot be explained by intellectual disabilities or global developmental delays (APA, 2013). The DSM-5 also indicates three levels of autism based on how much support is needed, where level 3 includes populations that require substantial support because of the lack of verbal and nonverbal
\end{abstract}


communication skills.

Kasari et al. (1990) suggests that children with ASD experience delays in the development of eye contact, gestures, body language, as used in non-verbal language outside the development of verbal language and affecting other general developments. Despite the absence of cure for children in the autism spectrum, the importance of variation in intervention in children with ASD at all ages is expressed by Spain et al. (2017) in a Cochrane Review on family therapy for children with ASD. The diversity of therapies is intended to reduce or ameliorate the symptoms and effects of ASD, in order to support children with ASD and those around them to improve their abilities.

Among the popular interventions, the method of putting forward play as a learning tool, coupled with structured instruction and home-based approach (Early Start Denver Model, otherwise known as ESDM) is one of the scientifically proven effective interventions for young children with ASD (APA, 2012). Another successful method is LEAP (Learning Experiences and Alternative Programs for Preschoolers and Their Parents) which involve children with ASD at an early age with a group of children with different developmental types in the classroom, so these children can adapt other children's behaviors through interaction and play process (APA, 2012).

\section{Music Therapy}

Music therapy is defined by the World Federation of Music Therapy (2014) as "the professional use of music and its elements as an intervention in the medical, educational ... environment to optimize the quality of life". In accordance with developmental psychology, playing (Winnicott, 1971) is an important stage in the process of music therapy because through this process a client learns to get to know about himself and his surroundings, and hence the development. Although music therapists may apply various methods according to the client population, age group, and relevant cultural background, music therapists with ASD children usually employ child-centered approaches, such as following the child's lead, offering choices, and presenting models.

Non-verbal interactions in music therapy are described as early parent-infant interactions, as coined by Stern (1985) as 'affect attunement'. The mother's response to her baby's nonverbal language will be the learning of the baby to learn her world. Stern (1985) also suggests that a mother allows her baby's development by slightly perform misattuning to her. To enable this to happen, three aspects need to be addressed: the parent's ability to identify the baby's emotional state, the parental ability to display behaviors relevant to the behaviors that the baby is performing, and the baby's ability to see the parent's response as something related to the feelings of their original experience (Stern, 1985, p.139).

The spontaneous movement of infants is greatly provoked by human nature to engage in emotional communication with others, or often known as 'communicative musicality' (Trevarthen\& Malloch, 2000). The term introduced by Stern (1985), 'affect attunement', consists of a set of musical elements to match the quality of interaction. The elements include: absolute intensity, contour intensity, temporal pulse, rhythm, duration, and shape (Stern, 1985, p.146). Trevarthen and Aitken (2001) claim that 'intersubjectivity' is shown in the way a mother responds to her baby's emotions and vice versa. A mutual coherence is built through this interaction, and it ultimately leads to the development of attachment (Trevarthen and Aitken, 2001, p.9).

\section{Music Therapy and Children with Autism}

A child's progress in therapy is assessed from their development. Juliette Alvin (1978) pioneered the practice of music therapy with ASD children in England in the 1960s. Furthermore, the practice of music therapy with this population evolved according to the advent of music therapy by using improvisational approaches initiated by Paul Nordoff and Clive Robins (1977). A meta-analysis by Whipple (2004) suggests that musical intervention, regardless of its use and purpose, has a significant impact on children and adolescents with ASD. Not only as an intervention, has music therapy also played a role as part of general assessment for children with ASD (Wigram and Gold, 2005).

In Indonesia, the form of music therapy with ASD, such as classical music therapy study conducted by Firdausiyah and Widajati (2013), follows procedures which is different from the ones such as defined by the WFMT. One of the factors of this difference is related to the perspective of ASD condition which focuses on behavioral aspects. Secondly, there is a general view that music therapy activities are emphasized on the listening (passive) activities. In addition, this study does not involve qualified music therapists who have undergone specific training. Nevertheless, interventions with classical music performed by Firdausiyah and Widajati (2013) show a positive result in decreasing hyperactive behavior of children with autism. This implies a relationship between music and its elements with ASD children.

Another consideration why music and its elements are considered suitable for this population is related to the characteristics of musical activities that can be perceived as a multi-sensory experience (Reschke-Hernandez, 2011, p.176). This is supported by the discovery by Michael Thaut (1988), the founder of Neurologic Music Therapy (NMT), regarding ASD's excellent musical ability and profound interest in music. As a means of expression, communication, and interaction, music is a medium that is easy to use by children compared to other mediums (Trevarthen, 2002). These documented findings motivate the development of practice and research of music therapy with ASD populations. Tony Wigram (2004) describes the practice of music therapy with ASD children in a book on the use of improvisational techniques in music therapy. To date, music therapy with 
ASD children (especially in developed countries) as a form of psychosocial intervention has been conducted individually, in groups, or involving families (Oldfield, 1993; Thompson, McFerran\& Gold, 2014).

\section{Musical Improvisation as a Technique in Music Therapy for Children with Autism}

In music practice, the views of free improvisation vary from simple, regular musical activities, to very complicated musical activities (Bailey, 1992, p.83-85). In fact, Wigram (2004) argues that improvisation can be a daunting thing for both trained musicians and people who have never attended music training, because although it seems like a simple and easy activity, improvisation is actually a complex process, even for professional musicians.

Improvisation is a form of technique that is often used in music therapy. Through improvisation, interpersonal and musical relationships between the therapist and the client are awakened. This concept is similar to the play theory coined by Winnicott (1971) where a child will develop when he has space to be creative and try new things through play. At a young age, children play and improvise naturally. But along with the development of age and awareness and the influence of the environment, they limit themselves and the ability to improvise will ultimately fade away.

Although the use of both active and receptive methods in music therapy has been found to have a good effect on the treatment of children with ASD, one of the most prominent and widely used active methods is improvisation. Considering one of the main purposes of music therapy is that children with ASD practice to initiate and maintain joint attention as well as improvisation in playing music into a suitable and useful medium (Wigram \& Gold, 2005). In a randomized controlled trial, Kim, Wigram, and Gold (2008) also found that the number and duration of eye contact in the improvisational music therapy sessions was significantly longer than in the playing sessions. The ability to maintain joint attention is an essential component that supports the development of other abilities in the areas of communication, social interaction, and language (Kim, Wigram, and Gold, 2008).

Wigram defines clinical improvisation as "the use of musical improvisation in an established trust and support environment to meet client needs" (2004, p.37). According to Trevarthen and Malloch (2000), clinical improvisation is inspired by the way a mother responds to her baby's actions and/or sounds emotionally, or as a term coined by Stern of 'affect attunement' (1985). This process is in line with the therapist-client's music interaction in music therapy settings, where the word 'attunement' implies a more flexible nature than pure imitation or mirroring. Two contrasting elements in improvisation, namely regularity in predictable structure and spontaneity, make this technique becomes effective for use in music therapy sessions with ASD children (Oldfield, 2006; Wigram, 2002).

\section{AIMS AND OBJECTIVES}

Although music therapy research, particularly in the area of treatment of children with autism and their families, has taken place in many developed countries, similar research has not been done in Indonesia. As a young discipline in this country, music therapy requires further investigation to define its role as a nonpharmacological intervention in the world of physical and mental health, as music therapy is an evidence-based practice that is inseparable from the local context.

In previous studies, it appears that music therapy has the potential in treating autism, especially through improvisation and the ability of music to serve as a nonverbal medium in therapy. The purpose of the study of music therapy with ASD children is to investigate the role of music therapy in the development of social communication skills of this population. Research questions explored within this research can be summarized as follows:

1. What is the role of music therapy in improving the communication and interaction of ASD children?

2. What is the musical process in music therapy in promoting social interaction between therapist and ASD children?

The present study is expected to provide an example of music therapy practice in ASD population in Indonesia and serve as a pilot study for further investigation, which may determine whether music therapy should be recommended as an intervention in the treatment of autism in this country.

\section{METHOD}

This is a single case study that explores the qualitative aspects of communication and social interaction in the music therapy sessions of a child with autism over the span of a year. After the consent form was signed by child's parent, I began to record the sessions using a camcorder and evaluate the session each week. Excerpts discussed are chosen from significant moments in the session where an emergence or improvement in communication area was captured. Conclusions are drawn from the elaboration of both behavioral and musical changes analyzed in the frames.

\section{Case Study}

Client's Background

John was a 10-year-old boy, diagnosed with autism. He lived with his parents and had two older siblings. He went to a regular kindergarten that accepts children with special needs. Both of his siblings attended a music school and played the piano. His mother referred him to music therapy, hoping that music could help John express 
his emotions. In January 2015, John began to have 45minute weekly music therapy sessions.

\section{Initial Observation and Assessment}

I came and observed John in his living room. It was an open space in the house that connects the side and backyard. There were sofas and an upright piano. During the first two weeks of assessment, he showed an interest in the guitar and small percussion instruments. He flitted from one instrument to another and struggled in sharing instruments with me. When I invited him to play the guitar together, he would mostly use his fingers to press down the strings as to stop my playing. When I played the piano, he would sometimes come and check out on what I was playing, but then withdrew and sat comfortably on the sofa. John occasionally vocalized, but most of them were not formed yet and unintelligible. Following the observation and assessment, I set goals for John which include express his emotions through music, improve communication skills, promote interaction, and improve listening skills and attention span.

\section{Sessions and Progress}

First stage: building rapport

We moved from the living room to therapy room in order to minimize distractions and give a clearer structure. In the first sessions I mostly attuned to John. Slowly he began to realize that I responded to what he did or the sounds he produced. During this moment, he would briefly look and/or smile at me, showing his emerging awareness of my presence. His number of eye contact slightly increased, but not long. Following my imitation or response to his action or voice, he occasionally presented meaningful smiles and laughter. Instead of stopping my guitar playing, John started to let me play. Sometimes he tapped the guitar while placing his ear close to the sound. Along with his growing interest in the strings instruments, particularly the guitar, he verbalised a few words such as "Plak!", which resembles the tapping sound, and something that sounds similar to his own name "John!" once he became very excited.

During this stage, John became slightly more flexible in activities that involve sharing instruments. His engagement in musical activities also developed. He expressed his enjoyment in music making and instrument preference by directly reaching out to the instrument, or sometimes directed my hand to take the instrument out of its case. While he previously had showed rigidity in handling the guitar, he now became more playful in doing it. He could stay in an activity with the guitar for 15 minutes, which also reflects a longer attention span.

Although sometimes John struggled to remain in the room for the whole session, he managed to stay calm and allowed me to enter his space. He would sit next to me at the piano while randomly pressing some keys or buttons. Becoming more aware of the structure, he would return to the therapy room after going to the toilet in the middle of the session. His frequency of looking and watching during the activities increased, especially when he was in control. Using drums with different sizes, I occasionally assessed his understanding of simple concept such as 'big' and 'small'. While most activities were improvisational and child-led, I tend to insert some therapist-directed activities to assess his reciprocity.

Second stage: increased response and playfulness

During this stage, John's communicative skills were increasingly developing. John began to make more interactions in the session, both musically and verbally (unintelligible words, up to 2-syllable words). His responses to music and verbal cue came out more spontaneously. In addition, John was beginning to effectively use the sign language he had previously learnt.

When I was playing the guitar (both strumming and tapping the guitar body), John started to vocalize and we took turns in vocalizing for five minutes, playing music together. During this time, he smiled and laughed a lot, swayed his body in rhythm, showing his delight.

John now welcomed physical contact such as highfive's and handshakes. He occasionally shared an instrument and allowed us to play it together. His way of playing shifted from a rigid way into trying out different ways to produce sounds. This flexibility was evident especially when he was playing the guitar. When he was engaged in our music making, he occasionally copied my way of strumming the guitar. It happened spontaneously without any prompt.

Furthermore, John also showed an interest in more instruments. He would listen to the music and look at the instrument I played (e.g. when I was improvising on the keyboard, he quietly looked at the keyboard as if he was observing the sound). Sometimes he would approach me and join in, other times he stayed where he was.

\section{Third stage: more responsiveness}

During the third stage, John's awareness of music therapy became more evident. Every time I came in for a session, he would readily grab the guitar, indicating the beginning of our session where I normally sing the Hello Song. He showed a lot of smiles when playing the guitar. His response to verbal instructions was increasingly quicker than before. For example, when I asked him to sit properly, he would instantly fix his posture. When I offered him a handshake, he spontaneously returned it. When I said goodbye, he responded by saying "Ba" ("Bye") and waving his hand.

John was looking through the window when I began to sing the Hello Song and strum the guitar to mark the beginning of our session. When I finished my first line, I paused and waved my hand. To my surprise, he looked at me and waved his hand as to respond to my greeting. This happened six times consecutively, although the intensity and length of his eye contact varied. 
His smiles and laughter appeared during his favorite musical activities and interactions. John's awareness of my presence also became more obvious. When looking at me during our interaction, he would look longer, almost as if he was trying to understand what lies behind our action. He began to use smile and glance to express his mischief, which indicates a new level of communication and understanding between us.Our interactions have grown into a meaningful connection.

When faced with unfamiliar tasks, there were a few times when John refused to respond to my invitation and shrieked "Aaahh!". When allowed time and space to adjust himself to the unfamiliarity, however, he would gradually negotiate and eventually execute the task. John generally appeared calm. He loved moving to rhythm, especially the firm and steady ones. He would normally rock his body and head while staring at the source of music, often in a long period. Although John could sit still and maintain his focus during an activity he was engaged in, he still occasionally took time to break, such as briefly galloping across the room, between the activities.

Since John has become more responsive, I began to invite him to exercise blowing to support his voice. The length, intensity and frequency of blowing were increasing each week. With improved eye contact, he started to imitate the shape of my lips when asked to say a word or syllable, although his volume was still too soft to produce a clear sound. The activity involved singing a song that has a section where I would start counting in five and visually showed him my fingers. This structure helped John anticipate when he needs to blow. Sometimes John would verbalize a lot during the session and I attuned to his verbal expression, which later on gave him a concept of turn-taking. Turn-taking also evolved during improvisation on the guitar and drum.

\section{DISCUSSION}

The extent of characteristics in the autism spectrum makes it difficult to determine a uniform intervention in the treatment of children with ASD. This study illustrates the use of music and its elements that involves complexity and simplicity at once to address an aspect in the development of children with ASD; their communication. Music has the potential to be an expressive tool for children with ASD to express choices, feelings, and responses when they are not ready to use their verbal means of communication. Elements in music, both offered structure and flexibility, attract children's attention and provoke eye contact, gestures, which become the predecessor of social interaction.

At the beginning, the therapist constantly attuned to the musical elements such as rhythm, melody, tempo, dynamics, contour, color, intensity initiated by the child, in order to raise the child's awareness of other's presence in the room. As soon as the child realized this, coupled with the appeal of music itself, interaction emerged. This continuous process results in the build of trust. Along with the development of trust and comfort in the relationship between the therapist and the child, comes the musical change. Through the process in improvisation, which is often associated with 'flow', the child begins to enjoy himself, but at the same time engaging in a joint activity. This is where communication and social experience take place.

Although the intervention was not long enough to show a significant improvement, the potential of the therapeutic use of music in ASD treatment can be seen, especially in children with autism who have a particular interest in music. This case is a preliminary study with limited time and number of samples. With the increasing availability of qualified music therapists in the country and the increasing awareness of music therapy, it is hoped that this study can be a starting point for further research with different methods, for example randomized controlled trials with a larger sample size to prove the effectiveness of music therapy interventions for ASD children, especially in the area of communication and social skills.

\section{REFERENCE}

Alvin, J. (1978). Music Therapy for the Autistic Child. London: Oxford University Press.

American Psychiatric Association. (2013). Diagnostic and Statistical Manual of Mental Disorders - DSM5.Washington DC: American Psychiatric Publishing.

Bailey, D. (1992). Improvisation - its Nature and Practice in Music. London: The British Library National Sound Archive.

Edwards, J. (2005). Developments and Issues in Music Therapy Research. In B. Wheeler (Ed.), Music therapy research (pp.20-32). Gilsum: Barcelona Publishers.

Firdausiyah, N. \&Widajati, W. (2013). TerapiMusikKlasikTerhadapPerilakuHiperaktifpadaA nakAutis. Jurnal Pendidikan Khusus. Vol.3.

Holck, U. (2011). Forskningimusikterapi - børn med enAutisme Spektrum Forstyrrelse. Tidsskrift for Dansk Musikterapi, 8(2), 27-35.

Kasari, C., Sigman, M., Munday, P. \&Yirmiya, N. (1990). Affective sharing in the context of joint attention interactions of normal, autistic, and mentally retarded children. Journal of Autism and Developmental Disorders, 20, 87-100.

Kim, J., Wigram, T. \& Gold, C. (2008). The effects of improvisational music therapy on joint behaviours in autistic children: a randomized controlled study. Journal of Autism Developmental Disorder, 38, 17581766.

Nordoff, P. \& Robbins, C. (1977). Creative Music Therapy. New York: John Day.

Oldfield, A. (1993). Music therapy with families. In M. Heal \& T. Wigram (Eds). Music Therapy in Health and Education. London: Jessica Kingsley Publishers.

Oldfield, A. (2006). Interactive Music Therapy in Child and Family Psychiatry. Clinical Practice, Research and Teaching. London: Jessica Kingsley Publishers. 
Papoušek, M. (2007). Communication in early infancy: An arena of intersubjective learning. Infant Behavior and Development, 30(2), 258-266.

Reschke-Hernandez, A. (2011). History of music therapy treatment interventions for children with autism. Journal of Music Therapy, 48, 169-207.

Spain, D., Sin, J., Paliokosta, E., Furuta, M., Prunty, J.E., Chalder, T., ... Happé, F.G. (2017). Family Therapy for Autism Spectrum Disorders. The Cochrane Library.Chichester: John Wiley \& Sons, Ltd.

Stern, D.N. (1985). The interpersonal world of the infant: a view from psychoanalysis and developmental psychology. New York: Basic Books.

Thaut, M. (1988). Measuring musical responsiveness in autistic children: A comparative analysis of improvised musical tone sequences of autistic, normal, and mentally retarded individuals. Journal of Autism and Developmental Disorders, 18(4), 561-571.

Thompson, G., McFerran, K. \& Gold, C. (2014). Family-centred music therapy to promote social engagement in young children with severe autism disorder: a randomized controlled study. Child: care, health and development, $40(6), 840-852$.

Trevarthen, C. (2002). Autism, symphaty of motives and music therapy. Enfance, 1, 95-131.

Trevarthen, C. \& Aitken, K.J. (2001). Infant intersubjectivity: Research, Theory, and Clinical Applications. Journal of Child Psychology Psychiatry, 42(1), 3-48.
Trevarthen, C. \& Malloch, S. (2000). The Dance of Wellbeing: Defining the Musical Therapeutic Effect, Nordisk Tidsskrift for Musikkterapi, 9(2), 3-17.

Wheeler, B. (Ed.). (2005). Music therapy research. Gilsum: Barcelona Publishers.

Whipple, J. (2004). Music in intervention for children and adolescents with autism: a meta-analysis. Journal of Music Therapy, 41, 90-106.

Wigram, T. (2002). Indications in music therapy: evidence from assessment that can identify the expectations of music therapy as a treatment for Autistic Spectrum Disorder (ASD): meeting the challenge of Evidence Based Practice. British Journal of Music Therapy, 16, 11-28.

Wigram, T. (2004). Improvisation - Methods and Techniques for Music Therapy, Clinicians, Educators and Students. London: Jessica Kingsley Publishers.

Wigram, T. \& Gold, C. (2005). Music therapy in the assessment and treatment of autistic spectrum disorder: clinical application and research evidence. Child: care, health and development, 32(5), 535-542.

Winnicott, D. (1971). Playing and Reality. London: Tavistock Publications.

www.edition.cnn.com/2017/08/08/health/autismmusic-therapystudy/index.html

www.wfmt.info/wpcontent/uploads/2014/05/ENGLISH-NEWWhat-is-music-therapy.pdf

www.who.int/mediacentre/factsheets/autism-spectrumdisorders/en/ 